\title{
CLUSTER SWAPPING
}

\author{
Juan C. Muzzio \\ National University of La Plata
}

\begin{abstract}
The investigation of globular cluster swapping in clusters of galaxies has resulted in some interesting theoretical findings and, at the same time, it offers a promising field for observers. Numerical simulations of galaxy clusters where the galaxies have swarms of test particles around them showed that, in addition to tidal stripping, tidal accretion plays an important role in the dynamical evolution of clusters of galaxies; it also turns out that, even in clusters where the gravitational field is dominated by a massive background, the galaxygalaxy attraction cannot be ignored when estimating the outcome of collisions. Cluster swapping is just an example of tidal accretion and, taking the globulars as probes of halo material, it might of fer an opportunity to observe some consequences of that effect; besides, although the difficulties look formidable at present, the study of globulars lost through tidal stripping is a possibility that should not be neglected. Tidal stripping and accretion processes are very sensitive to the ratio of galactic mass to total mass, so that observations related to the cluster swapping phenomena may provide a new means to investigate the missing mass problem.
\end{abstract}

\section{INTRODUCTION}

What happens to the globular cluster system of a galaxy that suffers an encounter with another galaxy? If the especial conditions required for a merger (low relative speed and small impact parameter) are not fullfilled, the system will be modified in three different ways: 1) through the capture of globulars from the other galaxy (cluster swapping), 2) through the loss of globulars to the other galaxy or to the intergalactic medium (tidal stripping), and 3 ) through changes in the orbits of the globulars that are kept by the system (Muzzio 1986a, hereafter Paper II).

Galaxies in groups and clusters suffer several encounters with other galaxies during their lifetime and, besides, they are also affected by their interaction with the cluster as a whole (Miller 1984); 
thus, it seems to be an interesting problem to investigate how their globular cluster systems evolve. Obviously, the stars of the galaxy will also be affected, but there are some advantages in concentrating in the evolution of the globular cluster systems because, although we can only study the integrated effect of the stellar light from distant galaxies, their globular clusters can be recognized as separate units. Accordingly while the interpretation of surface brighteness observations should take into account the stellar luminosity function and its possible variation, it is much simpler to interpret the observed surface densities of globulars. Besides, the possibility is open to the study of individual globulars and to the derivation of metallicities and radial velocities that, again, are not weighted by luminosity. Therefore, the study of globular cluster systems of galaxies that belong to clusters, in addition to be of interest by itself, may also provide useful information about the dynamical processes in clusters of galaxies.

\section{ENCOUNTERS OF GALAXIES}

The two most cited investigations about encounters of galaxies that do not lead to mergers are probably those of Richstone (1975) and of Dekel et al. (1980); interestingly enough, they did not take into account the possibility of swapping (or tidal accretion) and they just considered the changes due to tidal stripping and to the redistribution of the galactic material (but see Figure 4 of Dekel et a1. and its caption). The omission had an unfortunate consequence because, since most authors that later on made numerical simulations of clusters of galaxies used the results of those two investigations, the currently available cluster models (Merritt 1983; Miller 1983; Richstone and Malumuth 1983; Malumuth and Richstone 1984) do not include the effect of tidal accretion.

Alternatively, something can be learned about swaps and escapes from the work of Hills (1975), Hills and Fullerton (1980), Fullerton and Hills (1982) and Hills (1984) who investigated encounters between a binary star (or a star-planet system) and a stellar intruder. Since their experiments were planned for other purposes, they have some limitations for the study of the present subject, but they are well supplemented by the results of Muzzio, Rabolli and Martínez (1984) who used similar methods to investigate encounters of a galaxy-globular cluster pair with another galaxy. Several interesting facts were revealed by all these studies of collisions: a) essentially the same results are obtained using either the exact formula for the attraction law between two Schuster's spheres or the asymptotic approximation of Muzzio and Martínez (1982); b) the probabilities of swap and of escape are strongly dependent on the ratio between the two galactic masses but, provided that such ratio is not altered, the extension of the galaxies does not influence so much the results of the encounter; $c$ ) the probability of swap for head-on collisions increases steeply as the mass of the interloper is increased from one-half to twice the mass of the parent galaxy, but for larger mass ratios the change is much 
gentler up to, at least, a mass ratio of 64; the probability of swap is maximum for low relative velocities, and smoothly falls to zero as the speed increases; d) for low relative velocities the probability of escape for head-on encounters increases steeply as the mass ratio is increased from one-half to four and it virtually does not change for subsequent increases of that ratio; the probability of escape is low for small relative velocities and, for mass ratios up to four, decreases with increasing relative velocity immediately after reaching a maximum, while for larger mass ratios the probability remains at its maximum possible value of $100 \%$ over a large range of speeds and falls off only for very large relative velocities; e) the velocity parameter that best characterizes the collision is the ratio between the relative velocity of the galaxies at the time of impact and the orbital velocity of the globular; $f$ ) the highest probability of escape is obtained for a value of the impact parameter that increases with decreasing relative velocity; since the decrease of the probability of swap as the impact parameter increases is less steep, swaps may outnumber escapes for large impact parameters.

Muzzio et al. (1987, hereafter Paper IV) confirmed that the most adequate velocity parameter is the ratio of the relative velocity of the galaxies at the time of impact to the internal velocity of the galaxy and showed that, since the former velocity depends on the relative orbit of the galaxies which in turn depends on their mutual attraction, the forces that the galaxies exert on each other cannot be ignored even in cluster models dominated by a massive background.

\section{THE TEST PARTICLE APPROACH}

As indicated above, current models of galaxy clusters do not include the effect of tidal accretion and they are not suitable for the study of cluster swapping. Thus, in order to investigate in detail the swapping process, my coworkers and me (Muzzio, Martínez and Rabolli 1984, hereafter Paper I; Paper II; Muzzio 1986b, hereafter Paper III; Paper IV; Rabolli 1986) followed the approach pioneered by Forte et al. (1982) and we investigated models of clusters of galaxies where the galaxies had swarms of test particles around them. The advantage of using test particles, rather than full N-body codes (e.g. Carnevali et al. 1981), is that the gravitational fields of the galaxies and of the intracluster medium (ICM) are independent of the number of particles that belong to them; thus, there is no problem in having a galaxy with just a few test particles around it, so that one can use numbers of test particles proportional to the galactic mass and still be able to use a rather broad range of galactic masses. Besides, the computing time is approximately proportional to the number of galaxies times the number of galaxies plus test particles, and is shorter than in full $\mathrm{N}$-body codes where it is proportional to the square of the total number of particles. Finally, since the test particles do not interact with each other, relaxation effects pose no problem and one can use softening parameters that realistically 
represent the sizes of galaxies. The main disadvantage of the test particle approach is that galaxies are not self-consistent; therefore, the size, mass and gravitational field of a galaxy remain always the same, despite the changes that the galaxy suffers. Thus, our method cannot compete with the methods used by the authors cited above to simulate the evolution of whole clusters of galaxies; alternatively, it is able to provide a realistic cluster environment for the galaxies, and it is better than the other methods to investigate what happens to the galaxies themselves when they move within that environment.

Muzzio and Vergne (1986) have developed a new code that might offer the advantages of both the full $\mathrm{N}+$ body and the test particle methods without suffering their shortcomings. We use test particles but the masses and sizes of the galaxies and of the background are determined by the number and distribution of the particles that belong to them; in other words, the particles are individually regarded as massless, but when they act collectively they behave as massive particles whose distribution determines the general gravitational field. We have been able to preserve in this way the advantages of the test particle approach attaining, at the same time, such a high degree of self-consistency that we have been able to produce mergers with this new code.

\section{RESULTS}

Following Miller (1983), a given set of parameters and initial conditions will be referred to as a model and a particular realization of these parameters as a run. Between three and eight runs (obtained using different seed numbers for the random number generator) enter in each model.

All the models investigated thus far had adopted virialized initial conditions and, in view of the results of Miller (1983), there is an urgent need for models that start from non-equilibrium conditions. M.M. Vergne and myself have just begun to investigate such models and our, still very preliminary, results suggest that the cluster swapping processes are intensified during the pre-virialization stages of the cluster evolution.

\subsection{Number of galaxies and halo extension}

The number of galaxies in the Virgo-like cluster models of Papers I and III was limited to the 61 ("bright" models) or 111 ("faint" models) brightest, and most massive, galaxies. No significant differences arose between the results of both kinds of models, however, so that in other investigations "bright" models were preferred because they allow the inclusion of a larger number of test particles and, thus, the obtainment of more meaningful results. 
Since we suspected that the extension of the globular cluster systems could be a critical parameter of the tidal stripping and swapping processes, both "concentrated" and "extended" models were investigated in Papers I and III. The former imply a limiting radius of $420 \mathrm{kpc}$ for a galaxy like M87, while the corresponding value for the latter is $1050 \mathrm{kpc}$; these radii are proportional to the square root of the galactic masses. It is important to emphasize that these are limiting values and that, due to the steep radial distribution of globulars, the distances to the corresponding galactic centers are less than one-fourth of those radii for $91.5 \%$ of the globulars. The results of the models showed that the differences were much less important than we had suspected, and that reasonable inaccuracies in the extension of the more realistic "concentrated" models could not seriously affect their results. It is worth noting that Miller (1983) found that the mass-radius relationship was of little relevance for the tidal stripping results of his models.

\section{2 Influence of location}

Rabolli (1986) studied the influence of the galaxy location on the cluster swapping processes. She grouped the galaxies in radial distance bins according to two different criteria: a) their final position after $10^{10}$ years of dynamical evolution, and b) their timeaveraged radial distance over the same interval. The results obtained from the two different samplings are essentially the same, and in both cases it clearly turns out that galaxies in the central region of a cluster are strongly affected while galaxies outside that region suffer smaller or negligible effects.

Table I presents the global results obtained by Rabolli when the galaxies were grouped according to their final positions.

TABLE I

Influence of location on cluster swapping.

\begin{tabular}{|cccc|}
\hline $\begin{array}{c}\text { Radius } \\
(\mathrm{kpc})\end{array}$ & $\begin{array}{c}\text { Lost by capture } \\
(\%)\end{array}$ & $\begin{array}{c}\text { Lost by escape } \\
(\%)\end{array}$ & $\begin{array}{c}\text { Captured } \\
(\%)\end{array}$ \\
\hline $0.0-412.5$ & 23.3 & 21.3 & 24.2 \\
$412.5-825.0$ & 8.7 & 11.7 & 7.4 \\
$825.0-1650.0$ & 5.7 & 5.1 & 5.1 \\
$>1650.0$ & 0.0 & 1.2 & 0.6 \\
\hline
\end{tabular}

\subsection{Influence of the distribution of missing mass}

The results of Merritt (1983) and of Richstone and Malumuth (1983) suggested that the conclusions of Papers I, II and III could be altered if not all the cluster mass lay in the galaxies as had been assumed in our investigations. The suspicion was confirmed by the results of Paper IV which showed that when part of the cluster mass was assigned to a smoothly distributed background the previous conclusions were 
appreciably altered. If all the missing mass belongs to the background, the cluster swapping processes turn out to be irrelevant; under the more likely assumption that half of the total mass lies in the galaxies and half in the background, those processes are damped but they should still yield observable effects.

\subsection{Mass dependence and massive central galaxies}

One of the most interesting consequences of the cluster swapping process is its strong dependence on the masses of the galaxies involved, already noted by Forte et al. (1982). Figures 3 and 4 of Paper I present the histograms of the percentage of captured globulars versus the absolute magnitudes of the capturing galaxy, while Figure 5 of the same Paper presents the corresponding histograms for the globulars that are lost, due either to escape or to capture by other galaxies.

We emphasized in Paper I that even the least massive galaxies capture globulars, albeit a small percentage, but it is also important to emphasize that the percentage of lost clusters decreases with decreasing mass of the parent galaxy. The reason to insist on this point is that the idea exposed in the paper by Forte et al. (1982), that giant galaxies capture globular from dwarf galaxies, is still entertained by other researchers (cf. Harris 1986) and that idea is wrong according to the more recent evidence. Since the number of globulars captured by other galaxies decreases with decreasing mass of the parent galaxy, and since the total number of available globulars in a cluster like Virgo has a maximum near $M_{V}=-21$ mag and decreases rather sharply toward faint magnitudes (Figure 2 of Paper I), the number of swapped globulars that come from dwarf galaxies is, in fact, fairly small.

As a check, we examined the absolute magnitudes of the parent galaxies of the globulars captured by the massive central galaxies of all the runs used in Paper II. The mean difference between the visual absolute magnitude of the parent galaxy and that of the capturing galaxy turned out to be:

$$
\Delta \mathrm{M}_{\mathrm{v}}=-1.41 \pm 0.13 \mathrm{mag} \quad(\mathrm{N}=53, \sigma=0.92 \mathrm{mag})
$$

and only 2, out of 53, test particles came from galaxies more than 3 mag fainter than the capturing galaxy. This result strengthens our assertion that the bulk of the captured globulars does not come from dwarf galaxies. Cluster swapping is a game reserved to the big shots!

Another interesting question is the one raised by Forte et al. (1982): Could M87 have obtained its globular cluster system capturing them from other galaxies? Figure 1 shows the net (i.e., captures minus losses) percent variation of the number of globular clusters of the brightest galaxy versus the visual absolute magnitude of that galaxy for all the runs investigated thus far. Several facts 


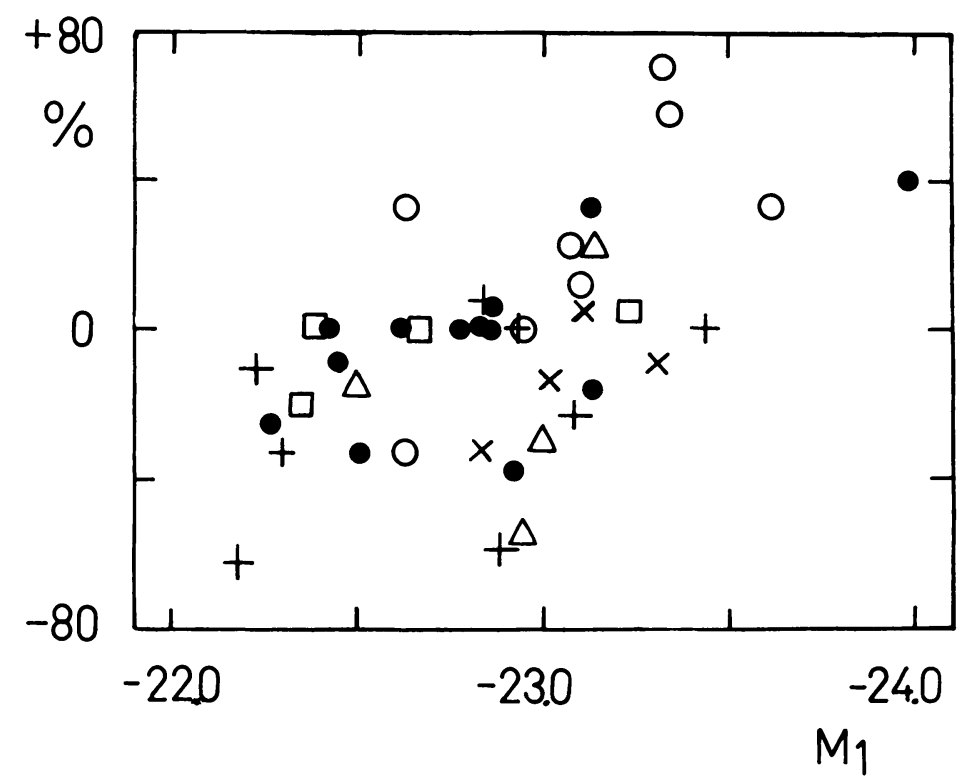

Fig. 1. Net percent variation of the number of globulars vs. absolute magnitude of first-ranked galaxies. Full circles: "concentrated" (Paper I and Rabolli 1986); open circles: "extended" (Paper I); plus signs: Paper II; triangles, crosses and squares: "a", "b" and "c", respectively, from Paper IV.

are clear from the Figure: 1) no model allows an increase larger than $70 \%$, or $40 \%$ if we consider only the "concentrated" models; 2) an absolute magnitude brighter than about -23.5 mag seems to rule out the possibility of a decrease, while galaxies fainter than $M_{V}=-22.5$ mag have no chance to increase their globular cluster population at the expense of others; notice that the masses implied by these absolute magnitudes are about $6.1 \quad 10^{13} \mathrm{M}_{\mathrm{O}}$ and $2.410^{13} \mathrm{M}_{\mathrm{O}}$, respectively; 3) the influence of the central location of the most massive galaxy is confirmed by the generally lower percentages yielded by the runs of Paper II as compared with the results of Paper I; 4) the influence of the galactic- to background-mass ratio is also confirmed by the smaller percentages (be they positive or negative) as one goes from model " $a$ ", through "b", to "c".

Unfortunately, we cannot yet be sure of which is the precise parameter that governs the net gain (or loss) of globulars because, for example, the magnitude difference between the first- and the secondranked galaxy yields a correlation similar to that shown in Figure 1. V.H. Dessaunet and myself are now preparing a new set of models that might help to clarify this situation; for the time being, it just seems possible for very massive galaxies to moderately increase their globular cluster population taking them from other galaxies. 


\subsection{Changes in the cluster distribution}

The relatively small net changes in the total population of a massive galaxy result from much larger gains and losses that partly compensate each other (Papers I and II); a central galaxy suffers quite a beating, and the structure of its globular cluster system becomes much more extended than that of an isolated galaxy. This scenario is strongly modified if most of the missing mass lies in the background and not in the galaxies (Paper IV) but if, as seems likely (Forman et al. 1985), the galaxies themselves contain an appreciable amount of missing mass, there will still be a substantial

modification of the distribution of globular clusters around a massive central galaxy.

The results of Papers I, II and IV showing that virtually all the outer halo (say, globulars beyond $210 \mathrm{kpc}$ from the center of the galaxy) is lost due to tidal interactions merely confirmed what one could have reasonably expected. What was rather surprising was the result that even globulars belonging to the inner parts of the halo are also lost in large quantities. Since the idea that tidal stripping only affects the outer parts of galaxies seems to be prevalent (e.g. Richstone and Malumuth 1983; Miller 1983; van den Bergh 1984) it is important to emphasize that, due to the central concentration of globulars (and matter in general) in galaxies, losses from the inner halo outweight those from the outer halo. If we consider the results of models "a" and " $b$ " from Paper IV, which do include a reasonable amount of background mass, we notice that for a galaxy like M87 the globulars lost from the region within a $50 \mathrm{kpc}$ radius almost double those lost from the rest of the galaxy; such radius corresponds to an angular radius of about $10^{\prime}$, i.e., well within the region where globulars have been studied in M87.

The globulars that are captured by a galaxy also tend to prefer the central regions, and their final radial distribution seems to be fairly independent of the original extension of the cluster systems (Paper I). The number of captures depends strongly on the ratio of galactic- to background-mass, however, and although model "a" of Paper IV still shows a substantial number of captured globulars, that number is very small for model "b".

The radial distribution of the globulars that remain with their parent galaxy becomes much more extended as a consequence of the tidal interactions. This result is strongly related to the amount of galaxy-galaxy interaction: it was negligible for the non-central galaxies of Paper I, very important for the central galaxies of Paper II, and varying from very important to completely negligible as the cluster dynamics become more and more dominated by the background (models "a", through "b", to "c" of Paper IV). 
4.6. Lost globulars

Following the ideas of Merritt (1983, 1984), Harris (1986) made the interesting suggestion that, as a result of tidal stripping, systems of globular clusters not related to any obvious central galaxy could sit at the center of clusters of galaxies. That possibility was investigated in detail in Paper III, and some related results were also given in Papers II and IV. The conclusion was that such systems are indeed possible but, unfortunately, the predicted surface density of globulars is very low and, therefore, very difficult to confirm observationally. The number of tramps decreases steadily as the background- to galacticmass ratio increases (Paper IV).

\section{OBSERVATIONAL POSSIBILITIES}

If there were observable effects of the cluster swapping processes, it would be possible to use the observational results to investigate some poorly known properties of clusters of galaxies (e.g. the distribution of missing mass) and to investigate individual globulars that had suffered the effect of those processes.

Following the ideas of Forte et al. (1982), Harris (1983) and van den Bergh (1984) used metallicity arguments to explore some consequences of cluster swapping. Unfortunately, if the bulk of the captured globulars does not come from dwarf galaxies but from galaxies only slightly less massive than the capturing galaxy, there is not much hope to get an observable metallicity difference. Nevertheless, the metallicity gradients within the globular cluster systems themselves (Strom et al. 1981) might allow the use of metallicity arguments in a different form: since the whole original outer halos are lost due to tidal stripping and replenished by cluster swapping with globulars that come mainly from the inner halos, we can expect that the metallicity of the outer halos will be higher for the galaxies that have suffered strong tidal stripping and accretion than for those that have had more peaceful lives. Accordingly, metallicity investigations of the globulars that lie beyond about $50 \mathrm{kpc}$ from the center of galaxies like M87 may provide an interesting observational test of the consequences of cluster swapping.

An even simpler test may be offered by the radial distribution of the globular clusters, because the results of Papers I and II and of Rabolli (1986) show that a galaxy located in the central region of a cluster should have a more extended distribution of globulars than a galaxy which lies farther from that center.

Harris (1986) derived revised radial distributions for the surface density of the globular clusters in both M87, located near the center of the Virgo cluster, and M49, located about $4^{\circ}$ from that center. If Harris's Figures 4 and 7 are superimposed and displaced 0.25 units along the vertical axis to account for the difference between the population 
of globulars in each galaxy, it turns out that for very large radii the surface density of globulars in M87 exceeds that in M49 at the same radius. The observational results are somewhat doubtful both for the innermost and the outermost regions, but it is interesting to note that, although small, the effect is in qualitative agreement with the theoretical predictions. We can only hope that the observational results will be improved in a near future, through the use of $C C D$ photometry for the inner regions (Grillmair et al. 1986), and of large field telescopes for the outer regions (Harris 1986).

It is very important to emphasize that a comparison between only two galaxies is not of much relevance, and that many systems should be observed and compared. The reason is that the theoretical predictions are of a statistical nature: they include averages of several runs, averages over several galaxies, averages over several globulars, and so on. Not only are the dispersions large, but the average results can be unapplicable to particular cases. For example, although we agree with Harris (1986) in that $\mathrm{M} 32$, wich has no globulars, is an excellent example of a dwarf galaxy that has been tidally stripped, we must emphasize that, as a satellite of M31, the situation of $M 32$ is very peculiar; in fact, as the results of Paper I show, dwarf galaxies are much less affected than giant galaxies by tidal stripping!

Since tidal accretion must be always accompanied by tidal stripping, the search for lost globulars seems to offer interesting possibilities, particularly considering that they might also offer a test for some theories of galaxy formation (e.g. van den Bergh 1980). Unfortunately, such a search presents formidable observational difficulties due to the very low surface density of the tramps: how to recognize them against the much larger surface density of foreground stars and background galaxies, how to be sure that they do not belong to the extended halo of a galaxy, and so on. Nevertheless, considering the interesting opportunities that their discovery would offer (systems of globular clusters without central galaxy, the end products of tidal stripping, and so on), and the rapid progress of the observational techniques, their study might soon become possible.

\section{ACKNOWLEDGEMENTS}

The technical assistance of Mrs. S.D. Abal de Rocha, M.C. Fanjul de Correbo and M.E. Mac Williams de Carstens is gratefully acknowledged. The author is a member of the Carrera del Investigador Científico del Consejo Nacional de Investigaciones Científicas y Técnicas de la República Argentina (CONICET). This work was supported by grants from the CONICET, the Comision de Investigaciones Cientificas de la Provincia de Buenos Aires and the Secretaría de Estado de Ciencia y Tecnología. 


\section{REFERENCES}

Carnevali, P., Cavaliere, A. and Santangelo, P. 1981 Astrophys. J. $249,449$.

Deke1, A., Lecar, M. and Shaham, J. 1980 Astrophys. J. 241, 946.

Forman, W., Jones, C. and De Faccio, M. 1985 in Proceedings of the ESO Workshop on the Virgo Cluster, 0.-G. Richter and B. Binggeli, eds., European Southern Observatory, Garching, p. 323.

Forte, J. C., Martinez, R. E. and Muzzio, J. C. 1982 Astron. J. 87,1465 .

Fullerton, L. W. and Hills, J. G. 1982 Astron. J. 87, 175.

Grillmair, C., Pritchet, C. and van den Bergh, S. 1986 Astron. J. 91,1328 .

Harris, W. E. 1983 Publ. Astron. Soc. Pacific 95, 21.

Harris, W. E. 1986 Astron. J. 91, 822.

Hills, J. G. 1975 Astron. J. 80, 809.

Hills, J. G. 1984 Astron. J. 89, 1559.

Hills, J. G. and Fullerton, L. W. 1980 Astron. J. 85, 1281.

Malumuth, E. M. and Richstone, D. 0. 1984 Astrophys. J. 276, 413.

Merritt, D. 1983 Astrophys. J. 264, 24.

Merritt, D. 1984 Astrophys. J. 276, 26.

Miller, G. E. 1983 Astrophys. J. 268, 495.

Miller, R. H. 1984 European Southern Observatory Scientific Preprint No. 343 .

Muzzio, J. C. 1986a Astrophys. J. 301, 23 (Paper II).

Muzzio, J. C. 1986b Astrophys. J., in press (Paper III).

Muzzio, J. C., Dessaunet, V. H. and Vergne, M. M. 1987 Astrophys. J. in press (Paper IV).

Muzzio, J. C. and Martinez, R. E. 1982 Obs. Astron. Univ. Nac. La Plata Serie Astron, XLI.

Muzzio, J. C., Martinez, R. E. and Rabolli, M. 1984 Astrophys. J. 285, 7 (Paper I).

Muzzio, J. C., Rabolli, M. and Martinez, R. E. 1984 unpublished.

Muzzio, J. C. and Vergne, M. M. 1986 in preparation.

Rabolli, M. 1986 Univ. Nac. La Plata Ph. D. Thesis in preparation.

Richstone, D. 0. 1975 Astrophys. J. 200, 535.

Richstone, D. O. and Malumuth, E. M. 1983 Astrophys. J. 268, 30 .

Strom, S. E., Forte, J. C., Harris, W. E., Strom, K. M., We1ls, D. C. and Smith, M. G. 1981 Astrophys. J. 245, 416.

van den Bergh, S. 1980 in Globular Clusters, D. Hanes and B. Madore, eds., Cambridge University Press, Cambridge, p. 175.

van den Bergh, S. 1984 Pub1. Astron. Soc. Pacific 96, 329. 


\section{DISCUSSION}

HARRIS: I think this simulation work is extremely interesting and something that we have needed to have for a long time. I would like to ask whether this work could be extended to small groups of galaxies as well as to richer environments like Coma. In the small groups of galaxies the encounter velocities are much lower, and you might end up with qualitatively different results. For example, in the fraction of escaped clusters or those that go through exchanges of any kind. Do you have any comments about the expected direction of the effects?

MUZZIO: I fully agree with you in that it will certainly be of interest to do models of small groups but since, as you say, the encounter velocities are lower, mergers may be more important there. Thus, I would rather investigate small groups with my new code, after having it thoroughly tested, because we will need self-consistency for that study.

OSTRIKER: I wonder if you will ever change the ratio of globular clusters to stars since when you find clusters are swapped, then stars will be swapped in the same ratio. Similarly the colors of the stars and the clusters are changed by the same amount. What are your views?

MUZZIO: I no longer entertain the idea of our original paper by Forte et al. (1982) that only the globulars are swapped; I now agree with you in that both globular clusters and stars are swapped and that is why I now prefer to call this effect tidal accretion rather than cluster swapping.

AGUILAR: The number of swapped clusters that you find seems very large. Simon White and I have studied tidal stripping using $\mathrm{N}$-body experiments with a self consistent target galaxy and found that particle swapping is negligible in most cases, and becomes important only when the encounter velocity at infinity is comparable to the internal rms velocity of the interacting galaxies. Since these encounters are very inelastic most of these encounters may lead to mergers in which case there is no swapping.

WALLERSTEIN: Is it possible to estimate what fraction of the 150 globular clusters in our Galaxy have been captured?

MUZZIO: Thank you very much for that question, because it allows me to emphasize once again that our results are average values. We did run some models of the local group, but the swapping activity turned out to be low there and dominated by random factors (say, the close passage of one galaxy near another), or, as Richstone and Malumuth (1983) very accurately put it, by "luck".

HANES: Your overlay of the Harris counts for M 87 and M 49 suggested 
that the outer parts of the M 87 cluster system is somewhat more distended. You should remember that the error bars are from size-of-sample statistics but that the counts in the outer parts are systematically very sensitive to the correct adoption of the local field contribution.

MUZZIO: I am fully aware of the uncertainty of the values in the outer regions and, besides, since our emphasis in on average values, a comparison of just two galaxies carrier little weight. I just wanted to show that $M 49$ and $M 87$ may be not identical and that the difference, although small, is in the sense predicted by the models; we certainly need more accurate observational results. 